\title{
Global Well-Posedness for Coupled System of mKdV Equations in Analytic Spaces
}

\author{
Khaled Zennir $\mathbb{D}^{1,2}$ Aissa Boukarou, ${ }^{3}$ and Rehab Nasser Alkhudhayr ${ }^{4}{ }^{4}$ \\ ${ }^{1}$ Department of Mathematics, College of Sciences and Arts, Qassim University, Ar Rass, Saudi Arabia \\ ${ }^{2}$ Laboratoire de Mathématiques Appliquées et de Modélisation, Université 8 Mai 1945 Guelma, B.P. 401, Guelma 24000, Algeria \\ ${ }^{3}$ Laboratoire de Mathématiques et Sciences Appliquées, Université de Ghardaia, Algeria \\ ${ }^{4}$ Department of Mathematics, College of Sciences, Qassim University, Buraydah, Saudi Arabia
}

Correspondence should be addressed to Rehab Nasser Alkhudhayr; rkhthaier@qu.edu.sa

Received 18 December 2020; Revised 22 January 2021; Accepted 11 February 2021; Published 5 March 2021

Academic Editor: Ioan Rasa

Copyright (C) 2021 Khaled Zennir et al. This is an open access article distributed under the Creative Commons Attribution License, which permits unrestricted use, distribution, and reproduction in any medium, provided the original work is properly cited.

The main result in this paper is to prove, in Bourgain type spaces, the existence of unique local solution to system of initial value problem described by integrable equations of modified Korteweg-de Vries ( $\mathrm{mKdV}$ ) by using linear and trilinear estimates, together with contraction mapping principle. Moreover, owing to the approximate conservation law, we prove the existence of global solution.

\section{Introduction and Main Results}

For an effective approach to solving problems arising in modern science and technology, one cannot do without researching nonlinear problems of mathematical physics. The rapid development of new technology and the emergence of its high speed allow researchers to build and consider increasingly complex multidimensional models describing various phenomena, which are modeled, as a rule, using nonlinear partial differential equations (systems). However, now it has become clear that without the development of analytical methods, it is impossible to get a complete idea of the essence of the phenomenon. Analytical methods provide not only a reliable tool for debugging and comparing various numerical methods but also sometimes anticipate some scientific discoveries, make it possible to study the properties of models, to detect the presence of certain effects as a result of the existence or nonexistence of objects (solutions) with the required properties. Therefore, at present, fundamental research is being intensively carried out aimed at proving theorems of existence, uniqueness, and regularity of solutions of nonlinear partial differential equations.
In the present paper, a coupled system of modified Korteweg-de Vries equations is considered as follows:

$$
\left\{\begin{array}{l}
\partial_{t} u+\partial_{x}^{3} u+\partial_{x}\left(u v^{2}\right)=0 \\
\partial_{t} v+\beta \partial_{x}^{3} v+\partial_{x}\left(u^{2} v\right)=0,(x, t) \in \mathbb{R}^{2}, 0<\beta<1, \\
u(x, 0)=u_{0}(x) \\
v(x, 0)=v_{0}(x)
\end{array}\right.
$$

The dynamics of solutions in the Korteweg-de Vries equations (KdV) and the modified Korteweg-de Vries equations (mKdV) are well studied due to the complete integrability of these equations (see [1-6]). For KdV equations, the studies date back to the 1970s, although some results have been obtained very recently (please see [7]). We extend the results in [7] and consider a coupled system of mKdV-type equations on the line in Equation (1).

For mKdV equations, many problems have been studied. It is proved that the $\mathrm{mKdV}$ equation is locally [8] and globally [9] well-posed in $H^{s}(\mathbb{T})$ for $s \geq 1 / 2$. Global wellposedness in $L^{2}(\mathbb{T})$ is shown in [10]. 
For $0<\beta<1$, the author in [7] proved that the IVP (Equation (1)) is locally well-posed for the given data $\left(u_{0}\right.$, $\left.v_{0}\right) \in H^{s}(\mathbb{R}) \times H^{s}(\mathbb{R}), s>-1 / 2$. Oh in [11] used the Fourier transform restriction norm method and proved that the next IVP

$$
\begin{array}{lc}
\partial_{t} u+\partial_{x}^{3} u+\partial_{x}\left(v^{2}\right)=0, & u(x, 0)=u_{0}(x), \\
\partial_{t} v+\beta \partial_{x}^{3} v+\partial_{x}(u v)=0, & v(x, 0)=v_{0}(x), 0<\beta<1,
\end{array}
$$

is locally well-posed for data with regularity $s \geq 0$.

For $\beta=1$, the system (Equation (1)) reduces to a special case of a broad class of nonlinear evolution equations considered by Ablowitz et al. [12] in the inverse scattering context. In this case, the well-posedness issues along with existence and stability of solitary waves for this system are widely studied in the literature, using the technique developed by Kenig et al. in $[13,14]$.

Well-posedness for the nonperiodic gKdV equation in spaces of analytic functions has been proved by Grujic and Kalisch [15].

A class of suitable analytic functions for our analysis is the analytic Gevrey class $G^{\delta, s}(\mathbb{R})=G^{\delta, s}$ introduced by Foias and Temam [16], defined as follows:

$$
G^{\delta, s}=\left\{f \in L^{2} ;\|f\|_{G^{\delta, s}}=\int_{\mathbb{R}} e^{2 \delta|\zeta|}\langle\zeta\rangle^{2 s}|f \wedge(\zeta)|^{2} d \zeta<\infty\right\}
$$

for $s \in \mathbb{R}$ and $\delta>0$ with $\langle\cdot\rangle=(1+|\cdot|)$. For $\delta=0$, the space $G^{\delta, s}$ coincides with the standard Sobolev space $H^{s}$. For all $0<\delta^{\prime}<\delta$ and $s, s^{\prime} \in \mathbb{R}$, we have

$$
G^{\delta, s} \subset G^{\delta^{\prime}, s^{\prime}} \text {, i.e., }\|f\|_{G^{\delta^{\prime}, s^{\prime}}} \leq c_{s, s}{ }^{\prime, \delta, \delta^{\prime}}\|f\|_{G^{\delta, s}},
$$

which is the embedding property of the Gevrey spaces.

New minimal conditions are used to show the local wellposedness of solution by using linear and trilinear estimates, together with contraction mapping principle. By imposing a more appropriate conditions with the help of the approximate conservation law, we obtain an unusual global existence result in Gevery spaces.

Proposition 1 (Paley-Wiener Theorem) [17]. Let $\delta>0, s \in \mathbb{R}$. Then, $f \in G^{\delta, s}$ if and only if it is the restriction to the real line of a function $F$ which is holomorphic in the strip $\{x+i y: x$, $y \in \mathbb{R},|y|<\delta\}$ and satisfies

$$
\sup _{|y|<\delta}\|F(x+i y)\|_{H_{x}^{s}}<\infty
$$

Remark 2. In the view of the Paley-Wiener Theorem, it is natural to take initial data in $G^{\delta, s}$, to obtain the best behavior of solution and may be extended to be globally in time. It means that given $\left(u_{0}, v_{0}\right) \in G^{\delta, s} \times G^{\delta, s}$ for some initial radius $\delta>0$, we then estimate the behavior of the radius of analyticity $\delta(T)$ over time.
The first main result on local well-posedness of Equation (1) in analytic spaces reads as follows.

Theorem 3. Let $\delta>0$ and $s>-1 / 2$. Then for any $\left(u_{0}, v_{0}\right) \in$ $G^{\delta, s} \times G^{\delta, s}$, there exists $T=T\left(\left\|\left(u_{0}, v_{0}\right)\right\|_{G^{\delta, s} \times G^{\delta, s}}\right)$ and unique solution $(u, v)$ of Equation (1) on $[0, T]$ such that

$$
(u, v) \in C\left([0, T], G^{\delta, s}\right) \times C\left([0, T], G^{\delta, s}\right) .
$$

Moreover, the solution depends on $\left(u_{0}, v_{0}\right)$, where

$$
T=\frac{1}{\left(16 C^{3}+16 C^{3}\left\|\left(u_{0}, v_{0}\right)\right\|_{G^{\delta, s} \times G^{\delta, s}}^{2}\right)^{1 / \varepsilon}} .
$$

Furthermore, the solution satisfies the following:

$$
\|(u, v)\|_{X_{\delta, s, b} \times X_{\delta, s, b}^{\beta}} \leq 2 C\left\|\left(u_{0}, v_{0}\right)\right\|_{G^{\delta, s} \times G^{\delta, s}}, \quad b=\frac{1}{2}+\varepsilon,
$$

with constant $C>0$ depending only on $s$ and $b$.

An effective method for studying lower bounds on the radius of analyticity, including this type of problem, was introduced in [18] for 1D Dirac-Klein-Gordon equations. It was applied in [19] to the modified Kawahara equation and in [20] to the nonperiodic $\mathrm{KdV}$ equation (for more details, please see [20-23]).

The second result for the problem (Equation(1)) is given in the next theorem.

Theorem 4. Let $s>-1 / 2,0<\beta<1$, and $\delta_{0}>0$. Assume that $\left(u_{0}, v_{0}\right) \in G^{\delta, s} \times G^{\delta, s}$, then the solution in Theorem 3 can be extended to be global in time and for any $T^{\prime}>0$, we have the following:

$$
(u, v) \in C\left(\left[0, T^{\prime}\right], G^{\delta\left(T^{\prime}\right), s}\right) \times C\left(\left[0, T^{\prime}\right], G^{\delta\left(T^{\prime}\right), s}\right),
$$

with

$$
\delta\left(T^{\prime}\right)=\min \left\{\delta_{0}, C_{1} T^{\prime-\left(2+\sigma_{0}\right)}\right\}
$$

where $\sigma_{0}>0$ can be taken arbitrarily small and $C_{1}>0$ is a constant depending on $w_{0}, \delta_{0}, s$, and $\sigma_{0}$.

The third result is Gevrey's temporal regularity of the unique solution obtained in the Theorem 3. A nonperiodic function $f(x)$ is the Gevrey class of order $r$, i.e., $f(x) \in G^{r}$, if there exists a constant $C>0$ such that

$$
\left|\partial_{x}^{l} f(x)\right| \leq C^{l+1}(l !)^{r} l=0,1,2, \cdots
$$

if $r=1 f(x)$ is analytic. 
Here, we will show that for $x \in \mathbb{R}$, for every $t \in[0, T]$ and $j, l \in\{0,1,2, \cdots\}$, there exist $C>0$ such that

$$
\begin{aligned}
& \left|\partial_{t}^{j} \partial_{x}^{l} u(x, t)\right| \leq C^{j+l+1}(j !)^{3}(l !), \\
& \left|\partial_{t}^{j} \partial_{x}^{l} v(x, t)\right| \leq C^{j+l+1}(j !)^{3}(l !),
\end{aligned}
$$

i.e., $(u(\cdot, t), v(\cdot, t)) \in G^{1}(\mathbb{R}) \times G^{1}(\mathbb{R})$ in spacial variable and $(u(x, \cdot), v(x, \cdot)) \in G^{3}([0, T]) \times G^{3}([0, T])$ in time variable. Also,

$$
\begin{aligned}
& \left|\partial_{t}^{j} \partial_{x}^{l} u(x, t)\right| \leq C^{j+l+1}(j !)^{d}(l !), \\
& \left|\partial_{t}^{j} \partial_{x}^{l} v(x, t)\right| \leq C^{j+l+1}(j !)^{d}(l !),
\end{aligned}
$$

where Equations (13) and (14) do not hold for $1 \leq d<3$.

Theorem 5. Let $s>-1 / 2,0<\beta<1$, and $\delta>0$. If $\left(u_{0}, v_{0}\right) \in$ $G^{\delta, s} \times G^{\delta, s}$, then the solution $(u, v) \in C\left([0, T], G^{\delta(T), s}\right) \times C([0$, $\left.T], G^{\delta(T), s}\right)$ given by Theorem 4 belongs to the Gevrey class $G^{3}([0, T]) \times G^{3}([0, T])$ in time variable. Furthermore, it is not belong to $G^{d}([0, T]) \times G^{d}([0, T]), 1 \leq d<3$ in $t$.

The proof of Theorem 5 is similar to that in [1].

The paper is organized as follows. In Section 2, we define the function spaces and linear and trilinear estimates. In Section 3, we prove Theorem 3, using the linear and trilinear estimates, together with contraction mapping principle. In Section 4, we prove the existence of fundamental approximate conservation law. In the last section, Theorem 4 will be proved using the approximate conservation law.

\section{Preliminary Tools and Analytic Function Spaces}

2.1. Function Spaces. We define the analytic Bourgain spaces related to the modified Korteweg-de Vries type equations. The completion of the Schwartz class $S\left(\mathbb{R}^{2}\right)$ is given by $X_{\delta, s, b}^{\beta}\left(\mathbb{R}^{2}\right)=X_{\delta, s, b}^{\beta}$, for $s, b \in \mathbb{R}, \delta>0$, subjected to the norm:

$$
\|w\|_{X_{\delta, s, b}^{\beta}}=\left(\int_{\mathbb{R}^{2}} e^{2 \delta|\zeta|}\langle\zeta\rangle^{2 s}\left\langle\eta-\beta \zeta^{3}\right\rangle^{2 b}|w \wedge(\zeta, \eta)|^{2} d \zeta d \eta\right)^{1 / 2}
$$

We often use without mention, the definition $X_{\delta, s, b}^{1}=$ $X_{\delta, s, b}$, where

$$
\|w\|_{X_{\delta, s, b}}=\left(\int_{\mathbb{R}^{2}} e^{2 \delta|\zeta|}\langle\zeta\rangle^{2 s}\left\langle\eta-\zeta^{3}\right\rangle^{2 b}|w \wedge(\zeta, \eta)|^{2} d \zeta d \eta\right)^{1 / 2}
$$

For any interval $I$, we define the localized spaces $X_{\delta, s, b}^{\beta}(\mathbb{R} \times I)=X_{\delta, s, b}^{\beta, I}$ with norm:

$$
\|w\|_{X_{\delta, s, b}^{\beta, L}}=\inf \left\{\|W\|_{X_{\delta, s, b}^{\beta}} ;\left.W\right|_{\mathbb{R} \times I}=w\right\} .
$$

2.2. Linear Estimates. We have the trilinear estimate (Equations (15) and (16)) defined in the analytic Bourgain spaces. Since the spaces $X_{\delta, s, b}^{\beta}$ is continuously embedded in $C\left([0, T], G^{\delta, s}\right)$, provided $b>1 / 2$.

Lemma 6. Let $b>1 / 2, s \in \mathbb{R}$, and $\delta>0$. Then, for all $T>0$, we have the following:

$$
X_{\delta, s, b} \hookrightarrow C\left([0, T], G^{\delta, s}\right) .
$$

Proof. First, we note that the operator $A$ defined by

$$
A w \wedge^{x}(\zeta, t)=e^{\delta|\zeta|} w \wedge^{x}(\zeta, t)
$$

satisfies

$$
\|w\|_{X_{\delta, s, b}^{\beta}}=\|A w\|_{X_{s, b}^{\beta}},\|w\|_{G^{\delta, s}}=\|A w\|_{H^{s}},
$$

where $X_{s, b}^{\beta}$ is introduced in [7]. We observe that $A w$ belongs to $C\left(\mathbb{R}, H^{s}\right)$ and for some $C>0$, we have the following:

$$
\|A w\|_{C\left(\mathbb{R}, H^{s}\right)} \leq C\|A w\|_{X_{s, b}^{\beta}}
$$

Thus, it follows that $w \in C\left([0, T], G^{\delta, s}\right)$ and

$$
\|w\|_{C\left([0, T], G^{\delta, s}\right)} \leq C\|w\|_{X_{\delta, s, b}^{\beta}} .
$$

Taking the Fourier transform with respect to $x$ of the Cauchy problems (Equation (1)), after an ordinary calculation, we localize in $t$ by using a cut-off function, satisfying $\psi \in C_{0}^{\infty}$, with $\psi=1$ in $[-1,1]$, supp $\psi \subset[-2,2]$, and $\psi_{T}(t)=$ $\psi(t / T)$. We consider the operator $\Lambda, \Gamma$ given by the following:

$$
\left\{\begin{array}{l}
\Lambda[u, v](t)=\psi(t) S(t) u_{0}-\psi_{T}(t) \int_{0}^{t} S(t-v) \partial_{x} F_{1}(v) d v, \\
\Gamma[u, v](t)=\psi(t) S_{\beta}(t) v_{0}-\psi_{T}(t) \int_{0}^{t} S_{\beta}(t-v) \partial_{x} F_{2}(v) d v,
\end{array}\right.
$$

where $S(t)=e^{-t \partial_{x}^{3}}$ and $S_{\beta}(t)=e^{-t \beta \partial_{x}^{3}}$ are the unitary groups associated with the linear problems.

The nonlinear terms defined by $F_{1}=\left(u v^{2}\right)$ and $F_{2}=\left(u^{2} \mathrm{v}\right)$ will be treated in the next lemmas. 
Lemma 7. Let $s, b \in \mathbb{R}$ and $\delta>0$. For some constant $C>0$, we have the following:

$$
\begin{gathered}
\left\|\psi(t) S(t) u_{0}\right\|_{X_{\delta, s, b}} \leq C\left\|u_{0}\right\|_{G^{\delta, s}}, \\
\left\|\psi(t) S_{\beta}(t) v_{0}\right\|_{X_{\delta, s, b}^{\beta}} \leq C\left\|v_{0}\right\|_{G^{\delta, s}},
\end{gathered}
$$

for all $u_{0}, v_{0} \in G^{\delta, s}$.

Proof. By definition, we have the following:

$$
\begin{aligned}
\psi(t) S_{\beta}(t) u_{0} & =C \psi(t) \int_{\mathbb{R}} e^{i\left(x \zeta+t \beta \zeta^{3}\right)} \widehat{u_{0}}(\zeta) d \zeta \\
& \left.=C \int_{\mathbb{R}^{2}} e^{i(x \zeta+t \eta)} \widehat{\psi}\left(\eta-\beta \zeta^{3}\right)\right) \widehat{u_{0}}(\zeta) d \zeta d \eta
\end{aligned}
$$

It follows that

$$
\begin{aligned}
\left\|\psi(t) S(t) u_{0}\right\|_{X_{\delta, s, b}^{\beta}}^{2}= & C \int_{\mathbb{R}^{2}} e^{2 \delta|\zeta|}(1+|\zeta|)^{2 s}\left(1+\left|\eta-\beta \zeta^{3}\right|\right)^{2 b} \\
& \cdot\left|\psi \wedge\left(\eta-\beta \zeta^{3}\right)\right|^{2}\left|u_{0} \wedge(\zeta)\right|^{2} d \zeta d \eta \\
= & C \int_{\mathbb{R}} e^{2 \delta|\zeta|}(1+|\zeta|)^{2 s}\left|u_{0} \wedge(\zeta)\right|^{2} \\
& \cdot\left(\int_{\mathbb{R}}\left|\psi \wedge\left(\eta-\beta \zeta^{3}\right)\right|^{2}\left(1+\left|\eta-\beta \zeta^{3}\right|\right)^{2 b} d \eta\right) d \zeta .
\end{aligned}
$$

Since $b>1 / 2$, we have the following:

$$
\begin{aligned}
\int_{\mathbb{R}} \mid \psi \wedge & \left.\left(\eta-\beta \zeta^{3}\right)\right|^{2}\left(1+\left|\eta-\beta \zeta^{3}\right|\right)^{2 b} d \eta \\
\leq & C \int_{\mathbb{R}}\left|\psi \wedge\left(\eta-\beta \zeta^{3}\right)\right|^{2} d \eta+C \int_{\mathbb{R}}\left|\psi \wedge\left(\eta-\beta \zeta^{3}\right)\right|^{2} \\
& \times\left(1+\left|\eta-\beta \zeta^{3}\right|\right)^{2 b} d \eta \leq C .
\end{aligned}
$$

Lemma 8. Let $s \in \mathbb{R},-1 / 2<b^{\prime} \leq 0 \leq b<b^{\prime}+1,0 \leq T \leq 1$, and $\delta>0$, then for some constant $C>0$, we have the following:

$$
\begin{aligned}
& \left\|\psi_{T}(t) \int_{0}^{t} S(t-v) \partial_{x} F_{1}(x, v) d v\right\|_{X_{\delta, s, b}} \leq C T^{1-b+b^{\prime}}\left\|\partial_{x} F_{1}\right\|_{X_{\delta, s, b}{ }^{\prime}}, \\
& \left\|\psi_{T}(t) \int_{0}^{t} S_{\beta}(t-v) \partial_{x} F_{2}(x, v) d v\right\|_{X_{\delta, s, b}^{\beta}} \leq C T^{1-b+b^{\prime}}\left\|\partial_{x} F_{2}\right\|_{X_{\delta, s, b^{\prime}}^{\beta}} .
\end{aligned}
$$

Proof. Define

$$
W=\psi_{T}(t) \int_{0}^{t} S_{\beta}(t-v) \partial_{x} F_{2}(x, v) d v
$$

We have, by Equation (19), the following:

$$
\begin{aligned}
A W \wedge^{x}(\zeta, t) & =\psi_{T}(t) \int_{0}^{t}\left(e^{-i(t-v) \beta \zeta^{3}}\right) e^{\delta|\zeta|} \partial_{x} F_{2} \wedge^{x}(\zeta, v) d v \\
& =\psi_{T}(t) \int_{0}^{t}\left[S_{\beta}(t-v)\left(\partial_{x} A F_{2}\right)\right] \wedge^{x}(\zeta, v) d v
\end{aligned}
$$

Thus,

$$
\|W\|_{X_{\delta, s, b}^{\beta}}=\|A W\|_{X_{s, b}^{\beta}}=\left\|\psi_{T}(t) \int_{0}^{t} S_{\beta}(t-v) \partial_{x} A F_{2}(x, v) d v\right\|_{X_{s, b}^{\beta}}
$$

Owing to Lemma 6 in [7], we get the following:

$$
\begin{aligned}
& \left\|\psi_{T}(t) \int_{0}^{t} S_{\beta}(t-v) \partial_{x} A F_{2}(x, v) d v\right\|_{X_{s, b}^{\beta}} \leq C T^{1-b+b^{\prime}}\left\|\partial_{x} A F_{2}\right\|_{X_{s, b^{\prime}}^{\beta}} \\
& =C T^{1-b+b^{\prime}}\left\|\partial_{x} F_{2}\right\|_{X_{\delta, s, b^{\prime}}^{\beta}} . \\
& \text { This completes the proof. }
\end{aligned}
$$

Lemma 9. Let $\Theta \in S(\mathbb{R})$ be a Schwartz function in time, $s \in \mathbb{R}$, and $\delta \geq 0$. If $-1 / 2<b \leq b^{\prime}<1 / 2$, then for any $T>0$, we have the following:

$$
\begin{gathered}
\left\|\Theta_{T}(t) w\right\|_{X_{\delta, s, b}} \leq C T^{b^{\prime}-b}\|w\|_{X_{\delta, s, b}{ }^{\prime}}, \\
\left\|\Theta_{T}(t) w\right\|_{X_{\delta, s, b}^{\beta}} \leq C T^{b^{\prime}-b}\|w\|_{X_{\delta, s, b^{\prime}}^{\beta}},
\end{gathered}
$$

where $C$ depends only on $b$ and $b^{\prime}$.

Proof. The proof of Lemma 9 for $\delta=0$ can be found in Lemma 13 of [14], for $\delta>0$ as one merely has to replace $w$ by $A w$, where the operator is defined in Equation (19).

Lemma 10 [20]. Let $s \in \mathbb{R}, \delta \geq 0,-1 / 2<b<1 / 2$, and $T>0$. Then, for any time interval $I \subset[0, T]$, we have the following:

$$
\begin{aligned}
& \left\|\chi_{I}(t) w\right\|_{X_{\delta, s, b}} \leq C\|w\|_{X_{\delta, s, b}^{T}}, \\
& \left\|\chi_{I}(t) w\right\|_{X_{\delta, s, b}^{\beta}} \leq C\|w\|_{X_{\delta, s, b}^{\beta, T}},
\end{aligned}
$$

where $\chi_{I}(t)$ is the characteristic function of I and $C$ depends only on $b$.

2.3. Trilinear Estimates. We have the trilinear estimate in the following lemmas. 
Lemma 11. Let $s>-1 / 2, \delta>0, b>1 / 2$, and $b^{\prime}$ be as in Lemma 8. Then,

$$
\begin{aligned}
& \left\|\partial_{x}\left(u v^{2}\right)\right\|_{X_{\delta, s, b}} \leq C\|u\|_{X_{\delta, s, b}}\|v\|_{X_{\delta, s, b}^{\beta}}^{2}, \\
& \left\|\partial_{x}\left(u^{2} v\right)\right\|_{X_{\delta, s, b^{\prime}}^{\beta}} \leq C\|u\|_{X_{\delta, s, b}}^{2}\|v\|_{X_{\delta, s, b}^{\beta}} .
\end{aligned}
$$

Proof. We observe, by considering the operator $A$ in (19), that

$$
\begin{aligned}
& e^{\delta|\zeta|} \widehat{u u v}=(2 \pi)^{-2} e^{\delta|\zeta|} \widehat{u} * \widehat{u} * \widehat{v} \leq(2 \pi)^{-2} \int_{\mathbb{R}^{4}} e^{\delta\left|\zeta-\zeta_{1}\right|} \widehat{u}\left(\zeta-\zeta_{1}, \eta\right.
\end{aligned}
$$

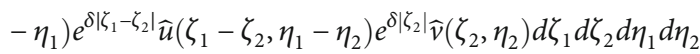

$$
\begin{aligned}
& =\widehat{A u A} A v \text {, }
\end{aligned}
$$

since $\delta|\zeta| \leq \delta\left|\zeta-\zeta_{1}\right|+\delta\left|\zeta_{1}-\zeta_{2}\right|+\delta\left|\zeta_{2}\right|$

Then,

$$
\begin{aligned}
\left\|\partial_{x}\left(u^{2} v\right)\right\|_{X_{\delta, s, b^{\prime}}^{\beta}} & =\left\|e^{\delta|\zeta|}\langle\zeta\rangle^{s}\left\langle\eta-\beta \zeta^{3}\right\rangle^{b} \partial_{x} \widehat{(u u v)}(\zeta, \eta)\right\|_{L_{\zeta, \eta}^{2}} \\
& \leq\left\|\partial_{x}(A u A u A v)\right\|_{X_{s, b^{\prime}}^{\beta}}
\end{aligned}
$$

Thanks to Proposition 2.3 of [7], for some $C>0$, we have the following:

$$
\left\|\partial_{x}(A u A u A v)\right\|_{X_{s, b^{\prime}}^{\beta}} \leq C\|A u\|_{X_{s, b}}^{2}\|A v\|_{X_{s, b}^{\beta}}=C\|u\|_{X_{\delta, s, b}}^{2}\|v\|_{X_{\delta, s, b}^{\beta}} .
$$

This completes the proof.

\section{Proof of Theorem 3}

3.1. Existence of Solution. We estimate terms in Equation (23). For this end, we define $B_{\delta, s, b}=X_{\delta, s, b} \times X_{\delta, s, b}^{\beta}$ and $N^{\delta, s}=$ $G^{\delta, s} \times G^{\delta, s}$, with norms $\|(u, v)\|_{B_{\delta, s, b}}=\max \left\{\|u\|_{X_{\delta, s, b}},\|v\|_{X_{\delta, s, b}^{\beta}}\right\}$ and similar for $N^{\delta, s}$

Lemma 12. Let $s>-1 / 2, \delta>0$, and $b>1 / 2$. Then, for all $\left(u_{0}\right.$, $\left.v_{0}\right) \in N^{\delta, s}$ and $0<T<1$, with some constant $C>0$, we have the following:

$$
\begin{aligned}
& \|(\Lambda[u, v], \Gamma[u, v])\|_{B_{\delta, s, b}} \leq C\left(\left\|\left(u, v_{0}\right)\right\|_{N^{\delta, s}}+T^{\mathcal{E}}\|(u, v)\|_{B_{\delta, s, b}}^{3}\right), \\
& \left\|\Lambda[u, v]-\Lambda\left[u^{*}, v^{*}\right], \Gamma[u, v]-\Gamma\left[u^{*}, v^{*}\right]\right\|_{B_{\delta, s, b}} \\
& \leq C T^{\varepsilon}\left\|\left(u-u^{*}, v-v^{*}\right)\right\|_{B_{\delta, s, b}} \\
& \quad \times\left(\|(u, v)\|_{B_{\delta, s, b}}^{2}+\|(u, v)\|_{B_{\delta, s, b}}\left\|\left(u^{*}, v^{*}\right)\right\|_{B_{\delta, s, b}}+\left\|\left(v^{*}, v^{*}\right)\right\|_{B_{\delta, s, b}}^{2}\right),
\end{aligned}
$$

for all $(u, v),\left(u^{*}, v^{*}\right) \in B_{\delta, s, b}$.
Proof. To prove estimate of Equation (39), we have the following:

$$
\begin{aligned}
& \|\Lambda[u, v]\|_{X_{\delta, s, b}} \leq C\left\|u_{0}\right\|_{G^{\delta, s}}+C T^{\mathcal{E}}\|u\|_{X_{\delta, s, b}}\|v\|_{X_{\delta, s, b}^{\beta}}^{2} \\
& \leq C\left\|\left(u_{0}, v_{0}\right)\right\|_{N^{\delta, s}}+C T^{\mathcal{\varepsilon}}\|(u, v)\|_{B_{\delta, s, b}}^{3}, \\
& \|\Gamma[u, v]\|_{X_{\delta, s, b}^{\beta}} \leq C\left\|v_{0}\right\|_{G^{\delta, s}}+C T^{\mathcal{\varepsilon}}\|u\|_{X_{\delta, s, b}}^{2}\|v\|_{X_{\delta, s, b}^{\beta}} \\
& \quad \leq C\left\|\left(u_{0}, v_{0}\right)\right\|_{N^{\delta, s}}+C T^{\varepsilon}\|(u, v)\|_{B_{\delta, s, b}}^{3} .
\end{aligned}
$$

Therefore, from Equations (41) and (42), we obtain the following:

$$
\|(\Lambda[u, v], \Gamma[u, v])\|_{B_{\delta, s, b}} \leq C\left(\left\|\left(u_{0}, v_{0}\right)\right\|_{N^{\delta, s}}+T^{\mathcal{E}}\|(u, v)\|_{B_{\delta, s, b}}^{3}\right) .
$$

For the estimate of Equation (40), we observe that

$$
\begin{aligned}
& \Lambda[u, v]-\Lambda\left[u^{*}, v^{*}\right]=\psi_{T}(t) \int_{0}^{t} S(t-v) \partial_{x}\left(u v^{2}-u^{*} v^{* 2}\right)(x, v) d v, \\
& \Gamma[u, v]-\Gamma\left[u^{*}, v^{*}\right]=\psi_{T}(t) \int_{0}^{t} S_{\beta}(t-v) \partial_{x}\left(u^{2} v-u^{* 2} v^{*}\right)(x, v) d v,
\end{aligned}
$$

where

$$
\begin{aligned}
& \omega=\partial_{x}\left(u^{2} v-u^{* 2} v^{*}\right)=\partial_{x}\left[v\left(u+u^{*}\right)\left(u-u^{*}\right)+u^{* 2}\left(v-v^{*}\right)\right] \\
& \omega^{\prime}=\partial_{x}\left(u v^{2}-u^{*} v^{* 2}\right)=\partial_{x}\left[u\left(v+v^{*}\right)\left(v-v^{*}\right)+v^{* 2}\left(u-u^{*}\right)\right] .
\end{aligned}
$$

We will show that $\Lambda \times \Gamma$ is a contraction on the ball $\mathbb{B}(0, R)$ to $\mathbb{B}(0, R)$, where $\mathbb{B}(0, R)$ is given in Equation (46).

Lemma 13. Let $s \geq-1 / 4, \delta>0$, and $b>1 / 2$. Then, for all $\left(u_{0}\right.$, $\left.v_{0}\right) \in N^{\delta, s}$, such that the map $\Lambda \times \Gamma: \mathbb{B}(0, R) \longrightarrow \mathbb{B}(0, R)$ is a contraction, where $\mathbb{B}(0, R)$ is given by the following:

$$
\mathbb{B}(0, R)=\left\{(u, v) \in B_{\delta, s, b} ;\|u, v\|_{B_{\delta, s, b}} \leq R\right\},
$$

with $R=2 C\left\|\left(u_{0}, v_{0}\right)\right\|_{N^{\delta, s}}$.

Proof. From Lemma 12, for all $(u, v) \in \mathbb{B}(0, R)$, we have the following:

$$
\begin{aligned}
& \|(\Lambda[u, v], \Gamma[u, v])\|_{B_{\delta, s, b}} \leq C\left\|\left(u_{0}, v_{0}\right)\right\|_{N^{\delta, s}}+C T^{\mathcal{E}}\|(u, v)\|_{B_{\delta, s, b}}^{3} \\
& \quad \leq \frac{R}{2}+C T^{\varepsilon} R^{3} .
\end{aligned}
$$
hence,

We choose $T$ sufficiently small such that $T^{\mathcal{E}} \leq 1 / 4 C R^{2}$;

$$
\|(\Lambda[u, v], \Gamma[u, v])\|_{B_{\delta, s, b}} \leq R, \forall(u, v) \in \mathbb{B}(0, R) .
$$


Thus, $\Lambda \times \Gamma$ maps $\mathbb{B}(0, R)$ into $\mathbb{B}(0, R)$, which is a contraction, since

$$
\begin{aligned}
& \|\left(\Lambda[u, v]-\Lambda\left[u^{*}, v^{*}\right], \Gamma[u, v]-\Gamma\left[u^{*}, v^{*}\right] \|_{B_{\delta, s, b}}\right. \\
& \leq \quad C T^{\varepsilon}\left\|\left(u-u^{*}, v-v^{*}\right)\right\|_{B_{\delta, s, b}} \\
& \quad \times\left(\|(u, v)\|_{B_{\delta, s, b}}^{2}+\|(u, v)\|_{B_{\delta, s, b}}\left\|\left(u^{*}, v^{*}\right)\right\|_{B_{\delta, s, b}}+\left\|\left(v^{*}, v^{*}\right)\right\|_{B_{\delta, s, b}}^{2}\right), \\
& \leq 3 C T^{\varepsilon} R^{2}\left\|\left(u-u^{*}, v-v^{*}\right)\right\|_{B_{\sigma, \delta, s, b}} \leq \frac{3}{4}\left\|\left(u-u^{*}, v-v^{*}\right)\right\|_{B_{\sigma, \delta, s, b}},
\end{aligned}
$$

for all $(u, v) \in \mathbb{B}(0, R)$. Hence, $(\Lambda, \Gamma): \mathbb{B}(0, R) \longrightarrow \mathbb{B}(0, R)$ is a contraction.

3.2. The Uniqueness. Uniqueness of the solution in $C([0, T]$, $\left.G^{\delta, s}\right) \times C\left([0, T], G^{\delta, s}\right)$ can be proved by the following standard argument.

Suppose that $(u, v),\left(u^{*}, v^{*}\right) \in C\left([0, T], G^{\delta, s}\right) \times C([0, T]$, $\left.G^{\delta, s}\right)$ are solutions to Equation $(1)$ with $(v(\cdot, 0), u(\cdot, 0))=\left(v^{*}\right.$ $\left.(\cdot, 0), u^{*}(\cdot, 0)\right)$ in $G^{\delta, s} \times G^{\delta, s}$. Setting $\vartheta=u-u^{*}$ and $\omega=v-v^{*}$, we see that $\vartheta, \omega$ solves the Cauchy problem:

$$
\begin{array}{ll}
\partial_{t} \vartheta+\partial_{x}^{3} \vartheta+\partial_{x}\left(u v^{2}-u^{*} v^{2}\right)=0, & \vartheta(0)=0, \\
\partial_{t} \omega+\partial_{x}^{3} \omega+\partial_{x}\left(u^{2} v-u^{2} v^{*}\right)=0, & \omega(0)=0 .
\end{array}
$$

Thus, by Equation (50), we have the following:

$$
\begin{aligned}
\frac{1}{2} \partial_{t}\|\vartheta(t, \cdot)\|_{L^{2}}^{2} & =\frac{1}{2} \partial_{t} \int_{\mathbb{R}} \vartheta^{2}(t, x) d x=\int_{\mathbb{R}} \vartheta(t, x) \partial_{t} \vartheta(t, x) d x \\
& =-\int_{\mathbb{R}} \vartheta(t, x) \partial_{x}\left(u v^{2}-u^{*} v^{2}\right) d x=0
\end{aligned}
$$

since we have the following:

$$
\int_{\mathbb{R}} \vartheta(t, x) \partial_{x}^{3} \vartheta(t, x) d x=0
$$

Thanks to Equation (53), we have the following:

$$
\partial_{t}\|\vartheta(t, \cdot)\|_{L^{2}}^{2}=-2 \int_{\mathbb{R}} \vartheta(t, x) \partial_{x}\left[v^{2} \vartheta(t, x)\right] d x
$$

Integrating by parts of the last integral, we obtain the following:

$$
\partial_{t}\|\vartheta(t, \cdot)\|_{L^{2}}^{2}=-\int_{\mathbb{R}} \partial_{x} v^{2}(t, x) \vartheta^{2}(t, x) d x
$$

from which we deduce the inequality as follows:

$$
\left|\partial_{t}\|\vartheta(t, \cdot)\|_{L^{2}}^{2}\right| \leq\left\|\partial_{x} v^{2}\right\|_{L^{\infty}}\|\vartheta(t)\|_{L^{2}}^{2}
$$

Since $u, u^{*} \in C\left([0, T], G^{\delta, s}\right)$, we have that $u$ and $u^{*}$ are continuous in $t$ on the compact set $[0, T]$ and are $G^{\delta, s}$ in $x$. Thus, we can conclude that

$$
\left\|\partial_{x} v^{2}\right\|_{L^{\infty}} \leq c<\infty
$$

Therefore, from Equations (56) and (57), we obtain the differential inequality:

$$
\left|\partial_{t}\|\vartheta(t, \cdot)\|_{L^{2}}^{2}\right| \leq c\|\vartheta(t)\|_{L^{2}}^{2}, \quad 0 \leq t \leq T .
$$

Solving it gives the following:

$$
\|\vartheta(t)\|_{L^{2}}^{2} \leq e^{c}\|\vartheta(0)\|_{L^{2}}^{2}, \quad 0 \leq t \leq T .
$$

Since $\|\vartheta(0)\|_{L^{2}}^{2}=0$, from Equation (59), we obtain that $\vartheta(t)=0,0 \leq t \leq T$, or $u=u^{*}$.

Now by Equation (51), we have the following:

$$
\left|\partial_{t}\|\omega(t, \cdot)\|_{L^{2}}^{2}\right| \leq c\|\omega(t)\|_{L^{2}}^{2}, \quad 0 \leq t \leq T .
$$

Solving it gives the following:

$$
\|\omega(t)\|_{L^{2}}^{2} \leq e^{c}\|\omega(0)\|_{L^{2}}^{2}, \quad 0 \leq t \leq T .
$$

Since $\|\omega(0)\|_{L^{2}}^{2}=0$, from Equation (61), we obtain that $\omega(t)=0,0 \leq t \leq T$, or $v=v^{*}$.

3.3. Continuous Dependence of the Initial Data. To prove continuous dependence of the initial data, we will prove the following.

Lemma 14. Let $s>-1 / 2, \delta>0$, and $b>1 / 2$. Then, for all $\left(u_{0}\right.$, $\left.v_{0}\right),\left(u_{0}^{*}, v_{0}^{*}\right) \in N^{\delta, s}$, if $(u, v)$ and $\left(u^{*}, v^{*}\right)$ are two solutions to Equation (1) corresponding to initial data $\left(u_{0}, v_{0}\right)$ and $\left(u_{0}^{*}\right.$, $\left.v_{0}^{*}\right)$, we have the following:

$$
\left.\left\|\left(u-u^{*}, v-v^{*}\right)\right\|_{C\left([0, T], G^{\delta, s}\right.}\right)^{2} \leq 4 C_{0} C\left\|\left(u_{0}-u_{0}^{*}, v_{0}-v_{0}^{*}\right)\right\|_{N^{\delta, s}} .
$$

Proof. If $(u, v)$ and $\left(u^{*}, v^{*}\right)$ are two solutions to Equation (1), corresponding to initial data $\left(u_{0}, v_{0}\right)$ and $\left(u_{0}^{*}, v_{0}^{*}\right)$, we have from Lemma 6 as follows:

$$
\begin{gathered}
\left\|u-u^{*}\right\|_{C\left([0, T], G^{\delta, s}\right)} \leq C_{0}\left\|u-u^{*}\right\|_{X_{\delta, s, b}}, \\
\left\|v-v^{*}\right\|_{C\left([0, T], G^{\delta, s}\right)} \leq C_{0}\left\|v-v^{*}\right\|_{X_{\delta, s, b}^{\beta}} .
\end{gathered}
$$

By taking $(u, v),\left(u^{*}, v^{*}\right) \in \mathbb{B}(0, R)$ and $T^{\varepsilon} \leq 1 / 4 C R$, we have the following:

$$
\begin{aligned}
& \left\|u-u^{*}\right\|_{X_{\delta, s, b}} \leq C\left\|\left(u_{0}-u_{0}^{*}, v_{0}-v_{0}^{*}\right)\right\|_{N^{\delta, s}}+\frac{3}{4}\left\|\left(u-u^{*}, v-v^{*}\right)\right\|_{B_{\delta, s, b}}, \\
& \left\|v-v^{*}\right\|_{X_{\delta, s, b}^{\beta}} \leq C\left\|\left(u_{0}-u_{0}^{*}, v_{0}-v_{0}^{*}\right)\right\|_{N^{\delta, s}}+\frac{3}{4}\left\|\left(u-u^{*}, v-v^{*}\right)\right\|_{B_{\delta, s, b}} .
\end{aligned}
$$


Thus,

$$
\left\|\left(u-u^{*}, v-v^{*}\right)\right\|_{B_{\delta, s, b}} \leq 4 C\left\|\left(u_{0}-u_{0}^{*}, v_{0}-v_{0}^{*}\right)\right\|_{N^{\delta, s}} .
$$

Then,

$$
\left\|\left(u-u^{*}, v-v^{*}\right)\right\|_{C\left([0, T], G^{\delta, s}\right)^{2}} \leq 4 C_{0} C\left\|\left(u_{0}-u_{0}^{*}, v_{0}-v_{0}^{*}\right)\right\|_{N^{\delta, s}} .
$$

This completes the proof of Theorem 3.

\section{Approximate Conservation Law}

We have the following:

$$
\|(u, v)\|_{L^{2}}=\int_{\mathbb{R}}\left(u^{2}+v^{2}\right) d x,
$$

which is conserved for a solution $(u, v)$ of Equation (1). We are going to show an approximate conservation law for a solution to Equation (1) based on the conservation of the $L^{2}(\mathbb{R})$ norm of solution.

Theorem 15. Let $\kappa \in[0,1 / 2)$ and $0<T_{1}<T_{0}<1, T_{0}$ be as in Theorem 3 with $s=0$; there exist $b=1 / 2+\varepsilon$ and $C>0$, such that for any $\delta>0$ and any solution $(u, v) \in B_{\delta, 0, b}^{T_{0}}$ to the Cauchy problem (Equation (1)) on the time interval $\left[0, T_{1}\right]$, we have the estimate:

$$
\sup _{t \in\left[0, T_{1}\right]}\|(u(t), v(t))\|_{N^{\delta, 0}}^{2} \leq\|(u(0), v(0))\|_{N^{\delta, 0}}^{2}+C \delta^{\kappa}\|(u, v)\|_{B_{\delta, 0, b}}^{4} .
$$

Moreover, we have the following:

$$
\sup _{t \in\left[0, T_{1}\right]}\|u(t), v(t)\|_{N^{\delta, 0}}^{2} \leq\|(u(0), v(0))\|_{N^{\delta, 0}}^{2}+C \delta^{\kappa} \|\left(u(0), v(0) \|_{N^{\delta, 0}}^{4} .\right.
$$

We need the following estimate.
Lemma 16. Given $\kappa \in[0,-1 / 2)$, there exist $b=1 / 2+\varepsilon, C>0$, and $(u, v) \in B_{\delta, 0, b}$; we have the following:

$$
\left\|\left(G_{1}, G_{2}\right)\right\|_{B_{0, b-1}} \leq C \delta^{\kappa}\|(u, v)\|_{B_{\delta, 0, b}}^{3},
$$

where $G_{1}=\partial_{x}\left[(A u A v A v)-A\left(u v^{2}\right)\right], \quad G_{2}=\partial_{x}[(A u A u A v)-$ $\left.A\left(u^{2} v\right)\right]$, and the operator $A$ is given by Equation (19).

Proof. Let $L_{1}=(A u A v A v)-A\left(u v^{2}\right)$. Then,

$$
\left\|G_{1}\right\|_{X_{0, b-1}}=\left\|\frac{\zeta}{\left\langle\eta-\zeta^{3}\right\rangle} \widehat{1-b} \widehat{L_{1}}(\zeta, \eta)\right\|_{L_{\zeta_{\zeta}}^{2}}=\left(\int_{\mathbb{R}^{2}} \frac{|\zeta|^{2}}{\left\langle\eta-\zeta^{3}\right\rangle^{2(1-b)}}\left|L_{1} \wedge(\zeta, \eta)\right|^{2} d \zeta d \eta\right) .
$$

We shall calculate the Fourier transform of $L_{1}$ as follows:

$$
\begin{aligned}
& |\widehat{L}(\zeta, \eta)|=\left|(A \widehat{u A v A v})-A \widehat{\left(u v^{2}\right)}\right| \\
& =C\left|\left(e^{\delta|\zeta|} \hat{u} * e^{\delta|\xi|} \hat{v} * e^{\delta|\zeta|} \hat{v} *\right)(\zeta, \eta)-e^{\delta|\zeta|}(\widehat{u} * \widehat{v} * \widehat{v} *)(\zeta, \eta)\right|
\end{aligned}
$$

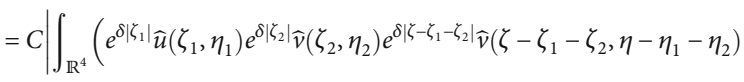

$$
\begin{aligned}
& \left.-e^{\delta|\xi|} \hat{u}\left(\zeta_{1}, \eta_{1}\right) \hat{v}\left(\zeta_{2}, \eta_{2}\right) \hat{v}\left(\zeta-\zeta_{1}-\zeta_{2}, \eta-\eta_{1}-\eta_{2}\right)\right) d \zeta_{1} d \zeta_{2} d \eta_{1} d \eta_{2} \mid \\
& \leq C \int_{\mathbb{R}^{4}}\left(e^{\delta\left|\zeta_{1}\right|} e^{\delta\left|\zeta_{2}\right|} e^{\delta\left|\zeta \zeta \zeta_{1}-\zeta_{2}\right|}-e^{\delta|\zeta|}\right) \\
& \times\left|\widehat{u}\left(\zeta_{1}, \eta_{1}\right) \hat{v}\left(\zeta_{2}, \eta_{2}\right) \hat{v}\left(\zeta-\zeta_{1}-\zeta_{2}, \eta-\eta_{1}-\eta_{2}\right)\right| d \zeta_{1} d \zeta_{2} d \eta_{1} d \eta_{2} .
\end{aligned}
$$

Now using Corollary 7.3 in [21], let $\theta \in[0,1]$; we have the following:

$$
\begin{aligned}
& e^{\delta\left|\zeta_{1}\right|} e^{\delta\left|\zeta_{2}\right|} e^{\delta\left|\zeta-\zeta_{1}-\zeta_{2}\right|}-e^{\delta|\zeta|} \\
& \quad \leq\left[4 \delta \frac{\left\langle\zeta-\zeta_{1}-\zeta_{2}\right\rangle\left\langle\zeta_{1}\right\rangle\left\langle\zeta_{2}\right\rangle}{\langle\zeta\rangle}\right]^{\theta} e^{\delta\left|\zeta_{1}\right|} e^{\delta\left|\zeta_{2}\right|} e^{\delta\left|\zeta-\zeta_{1}-\zeta_{2}\right|} .
\end{aligned}
$$

For $\kappa \in[0,1 / 2) \subset[0,1]$, one can see that

$$
\begin{aligned}
\left\|G_{1}\right\|_{X_{0, b-1}}^{2}= & \left\|\frac{\zeta}{\left\langle\eta-\zeta^{3}\right\rangle^{1-b}} \widehat{L_{1}}(\zeta, \eta)\right\|_{L_{\zeta, \eta}^{2}}^{2} \leq(C 4 \delta)^{2 \kappa} \int_{\mathbb{R}^{2}} \frac{|\zeta|^{2}}{\left\langle\eta-\zeta^{3}\right\rangle^{2(1-b)}} \\
& \cdot\left[\int_{\mathbb{R}^{4}}\left(\frac{\left\langle\zeta-\zeta_{1}-\zeta_{2}\right\rangle\left\langle\zeta_{1}\right\rangle\left\langle\zeta_{2}\right\rangle}{\langle\zeta\rangle}\right)^{\kappa} \times e^{\delta\left|\zeta_{1}\right|} e^{\delta\left|\zeta_{2}\right|} e^{\delta\left|\zeta \zeta-\zeta_{1}-\zeta_{2}\right|} \times\left|u \wedge\left(\zeta_{1}, \eta_{1}\right) v \wedge\left(\zeta_{2}, \eta_{2}\right) v \wedge\left(\zeta-\zeta_{1}-\zeta_{2}, \eta-\eta_{1}-\eta_{2}\right)\right| d \zeta_{1} d \zeta_{2} d \eta_{1} d \eta_{2}\right]^{2} d \zeta d \eta \\
= & C(4 \delta)^{2 \kappa}\left\|\frac{\zeta\langle\zeta\rangle^{-\kappa}}{\left\langle\eta-\zeta^{3}\right\rangle^{1-b}} \int_{\mathbb{R}^{4}} e^{\delta\left|\zeta_{1}\right|}\left\langle\zeta_{1}\right\rangle^{\kappa} \widehat{u}\left(\zeta_{1}, \eta_{1}\right) e^{\delta\left|\zeta \zeta_{2}\right|}\left\langle\zeta_{2}\right\rangle^{\kappa} \widehat{v}\left(\zeta_{2}, \eta_{2}\right) \times e^{\delta\left|\zeta-\zeta_{1}-\zeta_{2}\right|\left\langle\zeta-\zeta_{1}-\zeta_{2}\right\rangle^{\kappa} \hat{v}\left(\zeta-\zeta_{1}-\zeta_{2}, \eta-\eta_{1}-\eta_{2}\right) d \zeta_{1} d \zeta_{2} d \eta_{1} d \eta_{2}}\right\|_{L_{\zeta, \eta}^{2}}^{2} .
\end{aligned}
$$


Now by taking $s=-\kappa \in(-1 / 2,0]$, we obtain the following:

$$
\begin{aligned}
& \left\|G_{1}\right\|_{X_{0, b-1}} \leq C(4 \delta)^{\kappa}\left\|\frac{\zeta\langle\zeta\rangle^{s}}{\left\langle\eta-\zeta^{3}\right\rangle^{1-b}} \int_{\mathbb{R}^{4}} \frac{e^{\delta \mid \zeta_{1}} \mid \widehat{u}\left(\zeta_{1}, \eta_{1}\right)}{\left\langle\zeta_{1}\right\rangle^{s}} \frac{e^{\delta\left|\zeta_{2}\right|} \hat{v}\left(\zeta_{2}, \eta_{2}\right)}{\left\langle\zeta_{2}\right\rangle^{s}} \cdot \frac{e^{\delta\left|\zeta-\zeta_{1}-\zeta_{2}\right| \widehat{v}\left(\zeta-\zeta_{1}-\zeta_{2}, \eta-\eta_{1}-\eta_{2}\right)}}{\left\langle\zeta-\zeta_{1}-\zeta_{2}\right\rangle^{s}} d \zeta_{1} d \zeta_{2} d \eta_{1} d \eta_{2}\right\|_{L_{\zeta_{\zeta, n}}^{2}} \\
& \leq C(4 \delta)^{\kappa}\left\|\frac{\zeta\langle\zeta\rangle^{s}}{\left\langle\eta-\zeta^{3}\right\rangle^{1-b}} \int_{\mathbb{R}^{4}} \frac{e^{\delta\left|\zeta_{1}\right|}\left\langle\eta_{1}-\zeta_{1}^{3}\right\rangle^{b} \widehat{u}\left(\zeta_{1}, \eta_{1}\right)}{\left\langle\zeta_{1}\right\rangle^{s}\left\langle\eta_{1}-\zeta_{1}^{3}\right\rangle^{b}} \frac{\zeta_{\zeta_{2}} \mid\left\langle\eta_{2}-\beta \zeta_{2}^{3}\right\rangle^{b} \widehat{v}\left(\zeta_{2}, \eta_{2}\right)}{\left\langle\zeta_{2}\right\rangle^{s}\left\langle\eta_{2}-\beta \zeta_{2}^{3}\right\rangle^{b}} \times \frac{e^{\delta\left|\zeta-\zeta_{1}-\zeta_{2}\right|}\left\langle\eta-\eta_{1}-\eta_{2}-\beta\left(\zeta-\zeta_{1}-\zeta_{2}\right)^{3}\right\rangle^{b} \widehat{v}\left(\zeta-\zeta_{1}-\zeta_{2}, \eta-\eta_{1}-\eta_{2}\right)}{\left\langle\zeta-\zeta_{1}-\zeta_{2}\right\rangle^{s}\left\langle\eta-\eta_{1}-\eta_{2}-\beta\left(\zeta-\zeta_{1}-\zeta_{2}\right)^{3}\right\rangle^{b}} \times d \zeta_{1} d \zeta_{2} d \eta_{1} d \eta_{2}\right\|_{L_{\zeta_{n}}^{2}} .
\end{aligned}
$$

Then,

$$
\begin{aligned}
& \left\|G_{1}\right\|_{X_{0, b-1}} \leq C \delta^{\kappa}\|A u\|_{X_{0, b}}\|A v\|_{X_{0, b}^{\beta}}^{2}=C \delta^{\mathcal{K}}\|u\|_{X_{\delta, 0, b}}\|v\|_{X_{\delta, 0, b}^{\beta}}^{2} \\
& \quad \leq C \delta^{\mathcal{K}}\|(u, v)\|_{B_{\delta, 0, b}}^{3} .
\end{aligned}
$$

Now let $L_{2}=(A u A u A v)-A\left(u^{2} v\right)$. Then,

$$
\begin{aligned}
& \left\|G_{2}\right\|_{X_{0, b-1}} \leq C \delta^{\kappa}\|A u\|_{X_{0, b}}^{2}\|A v\|_{X_{0, b}^{\beta}}=C \delta^{\kappa}\|u\|_{X_{\delta, 0, b}}^{2}\|v\|_{X_{\delta, 0, b}^{\beta}} \\
& \quad \leq C \delta^{\kappa}\|(u, v)\|_{B_{\delta, 0, b}}^{3} .
\end{aligned}
$$

By Equations (76) and (77), we have the following:

$$
\left\|\left(G_{1}, G_{2}\right)\right\|_{B_{0, b-1}} \leq C \delta^{\kappa}\|(u, v)\|_{B_{\delta, 0, b}}^{3} .
$$

Proof (Theorem 15). Let $U(t, x)=A u(t, x), V(t, x)=A v(t, x)$ which are real-valued since the multiplier $A$ is even and $u, v$ are real-valued. Applying $A$ to Equation (1), we obtain the following:

$$
\begin{aligned}
& \partial_{t} U+\partial_{x}^{3} U+\partial_{x}\left(U V^{2}\right)=G_{1}, \\
& \partial_{t} U+\partial_{x}^{3} U+\partial_{x}\left(U^{2} V\right)=G_{2},
\end{aligned}
$$

where $G_{1}=\partial_{x}\left[(A u A v A v)-A\left(u v^{2}\right)\right]$ and $G_{2}=\partial_{x}[(A u A u A v)$ $\left.-A\left(u^{2} v\right)\right]$.

By multiplying both sides of Equation (79) by $U$ and Equation (80) by $V$ and integrating with respect to space variable, we get the following:

$$
\begin{aligned}
& \int_{\mathbb{R}} U \partial_{t} U d x+\int_{\mathbb{R}} U \partial_{x}^{3} U d x+\int_{\mathbb{R}} U \partial_{x}\left(U V^{2}\right) d x=\int_{\mathbb{R}} U G_{1} d x, \\
& \int_{\mathbb{R}} V \partial_{t} V d x+\int_{\mathbb{R}} V \partial_{x}^{3} V d x+\int_{\mathbb{R}} V \partial_{x}\left(U^{2} V\right) d x=\int_{\mathbb{R}} V G_{2} d x .
\end{aligned}
$$

Then,

$$
\begin{aligned}
& \int_{\mathbb{R}}\left(U \partial_{t} U+V \partial_{t} V\right) d x+\int_{\mathbb{R}}\left(U \partial_{x}^{3} U+V \partial_{x}^{3} V\right) d x+\int_{\mathbb{R}}\left[U \partial_{x}\left(U V^{2}\right)\right. \\
& \left.\quad+V \partial_{x}\left(U^{2} V\right)\right] d x=\int_{\mathbb{R}}\left(U G_{1}+V G_{2}\right) d x \\
& \int_{\mathbb{R}}\left(U \partial_{t} U+V \partial_{t} V\right) d x+\int_{\mathbb{R}} \partial_{x}\left(\partial_{x} U \partial_{x} U+\partial_{x} V \partial_{x} V\right) d x \\
& \quad+\int_{\mathbb{R}} \partial_{x}\left(U^{2} V^{2}\right) d x=\int_{\mathbb{R}}\left(U G_{1}+V G_{2}\right) d x .
\end{aligned}
$$

Noting that $\partial_{x}^{j} U(x, t) \longrightarrow 0$ as $|x| \longrightarrow \infty$ (see [20]), we use integration by parts to obtain the following:

$$
\frac{1}{2} \partial_{t} \int_{\mathbb{R}}\left(U^{2}+V^{2}\right) d x=\int_{\mathbb{R}}\left(U G_{1}+V G_{2}\right) d x .
$$

Integrating the last equality with respect to $t \in\left[0, T_{1}\right]$, we obtain the following:

$$
\begin{aligned}
& \int_{\mathbb{R}}\left(U^{2}\left(T_{1}, x\right)+V^{2}\left(T_{1}, x\right)\right) d x=\int_{\mathbb{R}}\left(U^{2}(0, x)+V^{2}(0, x)\right) d x \\
& \quad+2 \int_{\mathbb{R}^{2}} \chi_{\left[0, T_{1}\right]}(t)\left(U G_{1}+V G_{2}\right) d x d t .
\end{aligned}
$$

Thus,

$$
\begin{aligned}
& \left\|u\left(T_{1}\right)\right\|_{G^{\delta, 0}}^{2}+\left\|v\left(T_{1}\right)\right\|_{G^{\delta, 0}}^{2}=\|u(0)\|_{G^{\delta, 0}}^{2}+\|v(0)\|_{G^{\delta, 0}}^{2} \\
& \quad+2\left|\int_{\mathbb{R}^{2}} \chi_{\left[0, T_{1}\right]}(t)\left(U G_{1}+V G_{2}\right) d x d t\right| .
\end{aligned}
$$

By using Holder's inequality, Lemma 10, Lemma 9, and the fact that

$$
\frac{1}{2}<1-b<\frac{1}{2}, \frac{1}{2}<b-1<\frac{1}{2} .
$$


Since $b>1 / 2+\varepsilon$, we obtain the following:

$$
\begin{aligned}
\| \int_{\mathbb{R}^{2}} & \chi_{\left[0, T_{1}\right]}(t)\left(U G_{1}+V G_{2}\right) d x d t \mid \leq\left\|\chi_{\left[0, T_{1}\right]}(t) U\right\|_{X_{0,1-b}} \\
& \quad \times\left\|\chi_{\left[0, T_{1}\right]}(t) G_{1}\right\|_{X_{0, b-1}}+\left\|\chi_{\left[0, T_{1}\right]}(t) V\right\|_{X_{0,1-b}^{\beta}}\left\|\chi_{\left[0, T_{1}\right]}(t) G_{2}\right\|_{X_{0, b-1}^{\beta}} \\
\leq & C\|U\|_{X_{0,1-b}^{T_{1}}}\left\|G_{1}\right\|_{X_{0, b-1}^{T_{1}}}+C\|V\|_{X_{0,1-b}^{\beta, T_{1}}\left\|G_{2}\right\|_{X_{0, b-1}^{\beta, T}}^{\beta, 1}} \leq C\left\|\Theta_{T_{1}} U\right\|_{X_{0,1-b}}\left\|\Theta_{T_{1}} G_{1}\right\|_{X_{0, b-1}}+C\left\|\Theta_{T_{1}} V\right\|_{X_{0,1-b}^{\beta}}^{\beta}\left\|\Theta_{T_{1}} G_{2}\right\|_{X_{0, b-1}^{\beta}} \\
\leq & C\|U\|_{X_{0,1-b}}\left\|G_{1}\right\|_{X_{0, b-1}}+C\|V\|_{X_{0,1-b}^{\beta}}^{\beta}\left\|G_{2}\right\|_{X_{0, b-1}^{\beta}},
\end{aligned}
$$

where $\Theta_{T_{1}}=1$ for $t \in\left[0, T_{1}\right]$; we can conclude from Lemma 16:

$$
\begin{gathered}
\left|\int_{\mathbb{R}^{2}} \chi_{\left[0, T_{1}\right]}(t)\left(U G_{1}+V G_{2}\right) d x d t\right| \leq C\|U\|_{X_{0,1-b}}\left\|G_{1}\right\|_{X_{0, b-1}} \\
+C\|V\|_{X_{0,1-b}^{\beta}}\left\|G_{2}\right\|_{X_{0, b-1}^{\beta}} \leq C \delta^{\kappa}\|u\|_{X_{\delta, 0, b}}^{2}\|v\|_{X_{\delta, 0, b}^{\beta}}^{2} \\
\quad+C \delta^{\kappa}\|u\|_{X_{\delta, 0, b}}^{2}\|v\|_{X_{\delta, 0, b}^{\beta}}^{2}=2 C \delta^{\kappa}\|u\|_{X_{\delta, 0, b}}^{2}\|v\|_{X_{\delta, 0, b}^{\beta}}^{2} \\
\leq 2 C \delta^{\kappa}\|(u, v)\|_{B_{\delta, 0, b}}^{4} .
\end{gathered}
$$

Therefore,

$$
\begin{gathered}
\left\|u\left(T_{1}\right)\right\|_{G^{\delta, 0}}^{2}+\left\|v\left(T_{1}\right)\right\|_{G^{\delta, 0}}^{2} \leq\|u(0)\|_{G^{\delta, 0}}^{2}+\|v(0)\|_{G^{\delta, 0}}^{2}+2 C \delta^{\kappa}\|(u, v)\|_{B_{\delta, 0, b}}^{4} \\
2\left\|\left(u\left(T_{1}\right), v\left(T_{1}\right)\right)\right\|_{N^{\delta, 0}}^{2} \leq 2\|(u(0), v(0))\|_{N^{\delta, 0}}^{2}+2 C \delta^{\kappa}\|(u, v)\|_{B_{\delta, 0, b}}^{4} \\
\sup _{t \in\left[0, T_{1}\right]}\|(u(t), v(t))\|_{N^{\delta, 0}}^{2} \leq\|(u(0), v(0))\|_{N^{\delta, 0}}^{2}+C \delta^{\kappa}\|(u, v)\|_{B_{\delta, 0, b}}^{4} .
\end{gathered}
$$

Finally, by using Equation (8), we conclude that

$$
\sup _{t \in\left[0, T_{1}\right]}\|(u(t), v(t))\|_{N^{\delta, 0}}^{2} \leq\|(u(0), v(0))\|_{N^{\delta, 0}}^{2}+C \delta^{\kappa}\|(u(0), v(0))\|_{N^{\delta, 0}}^{4} .
$$

\section{Proof of Theorem 4}

Let $\delta_{0}>0, s>-1 / 2$, and $\kappa \in(0,1 / 2)$ be fixed, and $\left(u_{0}, v_{0}\right) \in$ $N^{\delta_{0}, s}$. Then, we have to prove that the solution $(u, v)$ of Equation (1) satisfies the following:

$$
(u, v) \in C\left(\left[0, T^{\prime}\right], G^{\delta\left(T^{\prime}\right), s}\right) \times C\left(\left[0, T^{\prime}\right], G^{\delta\left(T^{\prime}\right), s}\right),
$$

where

$$
\delta\left(T^{\prime}\right)=\min \left\{\delta_{0}, C_{1} T^{\prime-1 / \kappa}\right\}, \quad \text { for all } T^{\prime}>0,
$$

and $C_{1}>0$ is a constant depending on $u_{0}, v_{0}, \delta_{0}, s$, and $\kappa$. By
Theorem 3, there is a maximal time $T^{*}=T^{*}\left(u_{0}, v_{0}, \delta_{0}, s\right) \epsilon$ $(0, \infty]$, such that

$$
(u, v) \in C\left(\left[0, T^{*}\right], G^{\delta_{0}, s}\right) \times C\left(\left[0, T^{*}\right], G^{\delta_{0}, s}\right) .
$$

If $T^{*}=\infty$, it is done.

If $T^{*}<\infty$, as we assume henceforth, it remains to prove the following:

$$
(u, v) \in C\left(\left[0, T^{\prime}\right], G^{C_{1} T^{\prime}-1 / \kappa, s}\right) \times C\left(\left[0, T^{\prime}\right], G^{C_{1} T^{\prime}-1 / \kappa, s}\right), \quad \text { for all } T^{\prime} \geq T^{*} .
$$

5.1. The Case $S=0$. Fixed $T^{\prime} \geq T^{*}$; we will show that, for $\delta>0$, sufficiently small

$$
\begin{gathered}
\sup \\
t \in\left[0, T^{\prime}\right]\|(u(0), v(0))\|_{N \delta, 0}^{2} \leq 2\|(u(0), v(0))\|_{N^{\delta}, 0}^{2} .
\end{gathered}
$$

In this case, by Theorem 3 and Theorem 15 with

$$
T_{0}=\frac{1}{\left(16 C^{3}+32 C^{3}\|(u(0), v(0))\|_{N^{\delta 0,0}}^{2}\right)^{1 / \varepsilon}},
$$

the smallness conditions on $\delta$ will be

$$
\delta<\delta_{0}, \frac{2 T^{\prime}}{T_{0}} C \delta^{\kappa} 2^{2}\|(u(0), v(0))\|_{N^{\delta_{0}, 0}}^{2} \leq 1, \quad C>0 .
$$

Here, $C$ is the constant in Theorems 15 .

By induction, we check that

$\sup _{t \in\left[0, n T_{0}\right]}\|(u(t), v(t))\|_{N^{\delta, 0}}^{2} \leq\|(u(0), v(0))\|_{N^{\delta, 0}}^{2}+n C \delta^{\kappa} 2^{2}\|(u(0), v(0))\|_{N^{\delta_{0}, 0}}^{4}$,

$$
\sup _{t \in\left[0, n T_{0}\right]}\|(u(t), v(t))\|_{N^{\delta, 0}}^{2} \leq 2\|(u(0), v(0))\|_{N^{\delta_{0}, 0}}^{2},
$$

for $n \in\{1, \cdots, m+1\}$, where $m \in \mathbb{N}$ is chosen so that $T^{\prime} \in\left[m T_{0},(m+1) T_{0}\right)$. This $m$ does exist; by Theorem 3 and the definition of $T^{*}$, we have the following:

$$
T_{0}<\frac{1}{\left(16 C^{3}+16 C^{3}\|(u(0), v(0))\|_{N^{\delta, 0}, 0}^{2}\right)^{1 / \varepsilon}}<T^{*} \text {, hence } T_{0}<T^{\prime} .
$$

In the first step, we cover the interval $\left[0, T_{0}\right]$, and by Theorem 15, we have the following:

$$
\begin{aligned}
& \sup _{t \in\left[0, T_{0}\right]}\|(u(t), v(t))\|_{N^{\delta, 0}}^{2} \leq\|(u(0), v(0))\|_{N^{\delta, 0}}^{2} \\
& \quad+C \delta^{\kappa}\|(u(0), v(0))\|_{N^{\delta, 0}}^{4} \leq\|(u(0), v(0))\|_{N^{\delta, 0}}^{2} \\
& \quad+C \delta^{\kappa}\|(u(0), v(0))\|_{N^{\delta, 0}, 0}^{4}
\end{aligned}
$$


since $\delta \leq \delta_{0}$; we used the following:

$$
\|(u(0), v(0))\|_{N^{\delta, 0}} \leq\|(u(0), v(0))\|_{N^{\delta_{0}, 0}} .
$$

This satisfies Equation (98) for $n=1$, and Equation (99) is following and using again $\|(u(0), v(0))\|_{N^{\delta, 0}} \leq \|(u(0), v(0))$ $\|_{N^{\delta_{0}, 0}}$ as well as the following:

$$
C \delta^{\kappa}\|(u(0), v(0))\|_{N^{\delta_{0}, 0}}^{2} \leq 1
$$

Suppose now that Equations (98) and (99) hold for some $n \in\{1, \cdots, m\}$ and we prove that it holds for $n+1$, we estimate the following:

$$
\begin{aligned}
& \sup _{t \in\left[n T_{0},(n+1) T_{0}\right]}\|(u(t), v(t))\|_{N^{\delta, 0}}^{2} \leq\left\|w\left(n T_{0}\right)\right\|_{N^{\delta, 0}}^{2} \\
& +C \delta^{\kappa}\left\|\left(u\left(n T_{0}\right), v\left(n T_{0}\right)\right)\right\|_{N^{\delta, 0}}^{4} \leq\left\|\left(u\left(n T_{0} n T_{0}\right), v\left(n T_{0}\right)\right)\right\|_{N^{\delta, 0}}^{2} \\
& \quad+C \delta^{\kappa} 2^{2}\|(u(0), v(0))\|_{N^{\delta_{0}, 0}}^{4} \leq\|(u(0), v(0))\|_{N^{\delta, 0}}^{2} \\
& \quad+n C \delta^{\kappa} 2^{2}\|(u(0), v(0))\|_{N^{\delta_{0}, 0}}^{4}+C \delta^{\kappa} 2^{2}\|(u(0), v(0))\|_{N^{\delta_{0}, 0}}^{4}
\end{aligned}
$$

satisfying Equation (98) with $n$ replaced by $n+1$. To get Equation (99) with $n$ replaced by $n+1$, it is then enough to have the following:

$$
(n+1) C \delta^{\kappa} 2^{2}\|(u(0), v(0))\|_{N^{\delta_{0}, 0}}^{2} \leq 1,
$$

but this holds by Equation (97), since $n+1 \leq m+1 \leq T^{\prime} / T_{0}$ $+1<2 T^{\prime} / T_{0}$.

Finally, Equation (97) is satisfied for $\delta \in\left(0, \delta_{0}\right)$ such that

$$
\frac{2 T^{\prime}}{T_{0}} C \delta^{\kappa} 2^{2}\|(u(0), v(0))\|_{N^{\delta_{0}, 0}}^{2}=1
$$

Thus, $\delta=C_{1} T^{\prime-1 / \kappa}$, where

$C_{1}=\left(\frac{1}{C 2^{3}\|(u(0), v(0))\|_{N^{\delta_{0}, 0}}^{2}\left(16 C^{3}+32 C^{3}\|(u(0), v(0))\|_{N^{\delta_{0}, 0}}^{2}\right)^{1 / \varepsilon}}\right)^{1 / \kappa}$.

5.2. The General Case. For all $s$, by Equation (4), we have $u_{0}$, $v_{0} \in G^{\delta_{0}, s} \subset G^{\delta_{0} / 2,0}$.

For case $s=0$, it is proved that there is a $T_{2}>0$, such that

$$
\begin{aligned}
& (u, v) \in C\left(\left[0, T_{2}\right), G^{\delta_{0} / 2,0}\right) \times C\left(\left[0, T_{2}\right), G^{\delta_{0} / 2,0}\right), \\
& (u, v) \in C\left(\left[0, T^{\prime}\right], G^{2 \sigma T^{\prime-1 / \kappa}, 0}\right) \times C\left(\left[0, T^{\prime}\right], G^{2 \sigma T^{\prime-1 / \kappa}, 0}\right), \quad \text { for } T^{\prime} \geq T_{2},
\end{aligned}
$$

where $\sigma>0$ depends on $u_{0}, v_{0}, \delta_{0}$, and $\kappa$.
Applying again the embedding Equation (4), we now conclude that

$$
\begin{aligned}
& (u, v) \in C\left(\left[0, T_{2}\right), G^{\delta_{0} / 4, s}\right) \times C\left(\left[0, T_{2}\right), G^{\delta_{0} / 4, s}\right), \\
& (u, v) \in C\left(\left[0, T^{\prime}\right], G^{\sigma T^{\prime-1 / \kappa}, s}\right) \times C\left(\left[0, T^{\prime}\right], G^{\sigma T^{\prime-1 / \kappa}, s}\right), \quad \text { for } T^{\prime} \geq T_{2},
\end{aligned}
$$

which imply Equation (94). The proof of Theorem 4 is now completed.

\section{Data Availability}

No data were used in this study.

\section{Conflicts of Interest}

The authors declare that they have no competing interests.

\section{Acknowledgments}

Rehab Nasser Alkhudhayr would like to thank the Deanship of Scientific Research, Qassim University, for funding the publication of this project.

\section{References}

[1] A. Boukarou, K. Guerbati, K. Zennir, S. Alodhaibi, and S. Alkhalaf, "Well-posedness and time regularity for a system of modified Korteweg-de Vries-type equations in analytic Gevrey spaces," Mathematics, vol. 8, no. 809, pp. 1-16, 2020.

[2] A. Boukarou, K. Zennir, K. Guerbati, and S. G. Georgiev, "Well-posedness of the Cauchy problem of Ostrovsky equation in analytic Gevrey spaces and time regularity," Rendiconti del Circolo Matematico di Palermo Series, vol. 2, 2020.

[3] A. Boukarou, K. Zennir, K. Guerbati, and G. G. Svetlin, "Wellposedness and regularity of the fifth order KadomtsevPetviashvili I equation in the analytic Bourgain spaces," Annali Dell'universita'di Ferrara, vol. 66, no. 2, pp. 255-272, 2020.

[4] J. Bourgain, "Fourier transform restriction phenomena for certain lattice subsets and applications to nonlinear evolution equations," Geometric and Functional Analysis, vol. 3, no. 3, pp. 209-262, 1993.

[5] E. N. Pelinovsky and E. G. Shurgalina, "Two-soliton interaction within the framework of the modified Korteweg-de Vries equation," Radiophysics and Quantum Electronics, vol. 57, no. 10, pp. 737-744, 2015.

[6] A. V. Slyunyaev, "Dynamics of localized waves with large amplitude in a weakly dispersive medium with a quadratic and positive cubic nonlinearity," Journal of Experimental and Theoretical Physics, vol. 92, no. 3, pp. 529-534, 2001.

[7] X. Carvajal and M. Panthee, "Sharp well-posedness for a coupled system of mKdV-type equations," Journal of Evolution Equations, vol. 19, no. 4, pp. 1167-1197, 2019.

[8] C. E. Kenig, G. Ponce, and L. Vega, "A bilinear estimate with applications to the KdV equation," Journal of the American Mathematical Society, vol. 9, no. 2, pp. 573-603, 1996.

[9] J. Colliander, M. Keel, G. Staffilani, H. Takaoka, and T. Tao, "Sharp global well-posedness for KdV and modified KdV on 
$\mathbb{R}$ and $\mathbb{T}, " J o u r n a l$ of the American Mathematical Society, vol. 16, no. 3, pp. 705-749, 2003.

[10] T. Kappeler and P. Topalov, "Global well-posedness of mKdV in $L^{2}(\mathbb{T}, \mathbb{R})$," Communications in Partial Difference Equations, vol. 30, no. 1-3, pp. 435-449, 2005.

[11] T. Oh, "Diophantine conditions in well-posedness theory of coupled KdV-type systems: local theory," International Mathematics Research Notices, vol. 18, pp. 3516-3556, 2009.

[12] M. Ablowitz, D. Kaup, A. Newell, and H. Segur, "Nonlinear evolution equations of physical significance," Physical Review Letters, vol. 31, no. 2, pp. 125-127, 1973.

[13] C. E. Kenig, G. Ponce, and L. Vega, "On the ill-posedness of some canonical dispersive equations," Duke Mathematical Journal, vol. 106, no. 3, pp. 617-633, 2001.

[14] C. E. Kenig, G. Ponce, and L. Vega, "Well-posedness and scattering results for the generalized Korteweg-de Vries equation via the contraction principle," Communications on Pure and Applied Mathematics, vol. 46, no. 4, pp. 527-620, 1993.

[15] Z. Grujic and H. Kalisch, "Local well-posedness of the generalized Korteweg-de Vries equation in spaces of analytic functions," Differential and Integral Equations, vol. 15, no. 11, pp. 1325-1334, 2002.

[16] C. Foias and R. Temam, "Gevrey class regularity for the solutions of the Navier-Stokes equations," Journal of Functional Analysis, vol. 87, no. 2, pp. 359-369, 1989.

[17] Y. Katznelson, An Introduction to Harmonic Analysis, Dover Publications, New York, 1976.

[18] S. Selberg and A. Tesfahun, "On the radius of spatial analyticity for the 1d Dirac-Klein-Gordon equations," Journal of Differential Equations, vol. 259, no. 9, pp. 4732-4744, 2015.

[19] G. Petronilho and P. Leal da Silva, "On the radius of spatial analyticity for the modified Kawahara equation on the line," Mathematische Nachrichten, vol. 292, no. 9, pp. 2032-2047, 2019.

[20] S. Selberg and D. O. Silva, "Lower bounds on the radius of a spatial analyticity for the KdV equation," in Annales Henri Poincaré, pp. 1009-1023, Springer International Publishing, 2016.

[21] J. L. Bona, Z. Grujić, and H. Kalisch, “Algebraic lower bounds for the uniform radius of spatial analyticity for the generalized $\mathrm{KdV}$ equation," Annales de l'IHP Analyse Non Linéaire, vol. 22, no. 6, pp. 783-797, 2005.

[22] A. A. Himonas, H. Kalisch, and S. Selberg, "On persistence of spatial analyticity for the dispersion-generalized periodic $\mathrm{KdV}$ equation," Nonlinear Analysis: Real World Applications, vol. 30, pp. 35-48, 2017.

[23] S. Selberg and A. Tesfahun, "On the radius of spatial analyticity for the quartic generalized KdV equation," in Annales Henri Poincaré, vol. 18, no. 11pp. 3553-3564, Springer International Publishing, 2017. 